ELEN SOUZA ${ }^{1}$

ADRIANA YOSHIDA

Heloisa Peres ${ }^{1}$

Liliana de Angelo AndRade ${ }^{2}$

LUIS Otávio SARIAN'

SOPHIE DERCHAIN ${ }^{1}$

\title{
Preservação da fertilidade e dos ovários em mulheres com tumores anexiais benignos
}

\author{
Preservation of the fertility and the ovaries \\ in women with benign adnexal tumors
}

Artigo Original

Palavras-chave

Doenças dos anexos/cirurgia

Preservação de fertilidade

Preservação dos ovários

Neoplasias/cirurgia

Histerectomia

Keywords

Anexal diseases/surgery Fertility preservation

Ovarian conservation

Neoplasms/surgery

Hysterectomy

\section{Resumo}

OBJETIVO: Avaliar a preservação da fertilidade e dos ovários em mulheres submetidas à cirurgia por tumor anexial benigno. MÉTODOS: Para este estudo observacional com coleta prospectiva foram incluídas 206 mulheres operadas no CAISM-Unicamp de fevereiro de 2010 a janeiro de 2014. A preservação da fertilidade foi definida como tumorectomia ou anexectomia unilateral sem histerectomia em mulheres na pré-menopausa. A preservação ovariana foi considerada quando pelo menos um ovário ou parte dele foi preservado. RESULTADOS: Das 206 mulheres com tumores anexiais benignos, 120 (58\%) estavam na pré-menopausa e 86 (42\%) na pós-menopausa. Na pré-menopausa, foram encontrados $36(30 \%)$ tumores de células germinativas, 31 (26\%) neoplasias epiteliais e 11 (9\%) do cordão sexual e estroma. Na pós-menopausa foram identificados 35 (41\%) neoplasias epiteliais, 27 (31\%) do cordão sexual e estroma e $8(9 \%)$ de células germinativas. Entre as 36 mulheres com tumores ovarianos não neoplásicos, 21 (58\%) apresentavam endometriomas e $8(22 \%)$ cistos funcionais. Das 22 mulheres com tumores extra ovarianos, o leiomioma uterino foi o achado mais frequente (50\%). Entre as pacientes com $\leq 35$ anos, 26 (57\%) foram submetidas à tumorectomia e $18(39 \%)$ a anexectomia unilateral com preservação do útero e anexo contralateral. Mulheres com $\leq 35$ anos foram mais frequentemente operadas por laparoscopia que esteve associada a maior taxa de preservação de fertilidade quando comparada com a laparotomia $(p<0,01)$. Observou-se que 26 das pacientes submetidas à histerectomia com anexectomia (28\%) bilateral estavam na pré-menopausa. CONCLUSÕES: Embora se observe uma tendência em realizar apenas tumorectomia em mulheres com $\leq 35$ anos, uma proporção significativa de mulheres jovens ainda é submetida à anexectomia. Em mulheres entre 36 e 45 anos, apenas dois terços tiveram sua fertilidade preservada e $20 \%$ tiveram ambos os ovários removidos. No entanto, deve-se tentar preservar os ovários sempre que possível, sobretudo nas mulheres na pré-menopausa.

\section{Abstract}

PURPOSE: To evaluate the sparing of fertility and ovaries in women submitted to surgical treatment for benign adnexal tumors. METHODS: Between February 2010 and January 2014, 206 patients were included in this observational study as they were submitted to surgical treatment for benign ovarian tumors at CAISM, a tertiary hospital. Fertility sparing surgery was defined as tumorectomy or unilateral salpingoophorectomy without hysterectomy in premenopausal women. Preservation of the ovary occurred when at least one ovary or part of it was mantained. RESULTS: Of the 206 women with benign tumors, $120(58 \%)$ were premenopausal and $86(42 \%)$ were postmenopausal. There were $36(30 \%)$ ovarian germ cell tumors, 31 (26\%) epithelial neoplasms and 11 (9\%) sex-cord stromal tumors among premenopausal women. In the group of postmenopausal women, 35 (4 1\%) epithelial neoplasms, 27 (31\%) sex-cord stromal tumors and $8(9 \%)$ ovarian germ cell tumors were identified. Among 36 women with non-neoplastic ovarian tumors, 21 (58\%) had
Correspondênnia

Sophie Derchain Departamento de Ginecologia e Obstetrícia, da Faculdade de Ciências Médicas da Universidade Estadual de Campinas Rua Alexander Flemming, 101 - Cidade Universitária "Zeferino Vaz" Barão Geraldo Caixa Postal 6111 (EP: $13083-970$ Campinas (SP), Brasil

Recebido $6 / 10 / 2014$

Aceito com modificacōoses $28 / 11 / 2014$
Hospital da Mulher Professor Doutor Aristodemo Pinotti, Centro de Atenção Integral à Saúde da Mulher, Universidade Estadual de Campinas - UNICAMP - Campinas (SP), Brasil.

'Departamento de Ginecologia e Obstetrícia, Faculdade de Ciências Médicas, Universidade Estadual de Campinas - UNICAMP Campinas (SP), Brasil.

2Departamento de Anatomia Patológica, Faculdade de Ciências Médicas, Universidade Estadual de Campinas - UNICAMP Campinas (SP), Brasil.

Conflito de interesses: não há 
endometriomas and 8 (22\%) functional cysts. Among 22 women with extra-ovarian tumors, uterine leiomyomatosis was the most frequent finding (50\%). In the group of women who were $\leq 35$ years old, 26 (57\%) were treated by tumorectomy and $18(39 \%)$ were submitted to unilateral salpingoophorectomy with sparing of the uterus and the contralateral ovary. Women who were $\leq 35$ years old were more frequently operated by laparoscopy which was associated with a higher number of fertility sparing procedures when compared to laparotomy $(p<0.01)$. Twenty-six (28\%) women submitted to hysterectomy with bilateral salpingoophorectomy were premenopausal. CONCLUSION: Although there is a trend to perform only tumorectomy in women who are $\leq 35$ years old, a significant number of young women is still treated by salpingoophorectomy. Among 36- to 45-year-old women, only $70 \%$ had their fertility spared, while $20 \%$ had both ovaries removed. However, whenever possible, we must try to preserve the ovaries, mainly in premenopausal women.

\section{Introdução}

Lesões anexiais são comuns em mulheres de todas as idades. Estima-se que aproximadamente $10 \%$ da população feminina é submetida a cirurgias para avaliar massas anexiais em algum momento ${ }^{1}$. Cerca de $70 \%$ dos tumores ovarianos ocorrem em idade reprodutiva, com maior incidência entre 21 e 40 anos $^{2}$.

Os tumores ovarianos benignos mais frequentes na pré-menopausa são os teratomas maduros císticos ou cistos dermóides, que correspondem a $20 \%$ de todas as neoplasias ovarianas e têm pico de incidência em mulheres de 20 a 40 anos, sendo a maioria unilaterais, podendo ser bilaterais em $10 \% 3$. Na peri e pós menopausa, predominam os cistadenomas serosos, que correspondem a dois terços dos tumores ovarianos epiteliais benignos, com maior incidência entre 40 a 60 anos, sendo que podem ser bilaterais em $20 \%$ dos casos. Já os cistadenomas mucinosos compreendem $13 \%$ das neoplasias ovarianas epiteliais benignas. A média de idade de apresentação é de 50 anos e são tipicamente unilaterais $(95 \%)^{4}$.

$\mathrm{Na}$ abordagem cirúrgica de massas anexiais, a preservação de fertilidade e da função hormonal pode ser considerada, levando-se em conta o tipo histológico do tumor, idade, prole e estado menopausal da paciente. No menacme, essencialmente em mulheres com prole incompleta e tumores potencialmente bilaterais sincrônicos ou assincrônicos, a tumorectomia com preservação dos dois anexos deve ser priorizada ${ }^{5}$. Nas mulheres menopausadas, a remoção cirúrgica de ambos os anexos e do útero deve ser realizada 5 .

Se para as pacientes no menacme a necessidade de preservação da função ovariana é reconhecida, já que os riscos de menopausa cirúrgica precoce não se justificam no tratamento dos tumores benignos do ovário, na peri-menopausa surgem fatores que levam à perda precoce da função ovariana, como oportunidade de se realizar ooforectomia "profilática" para câncer, risco de nova abordagem cirúrgica devido a aparecimento de novos cistos ou tumores e presença de irregularidades menstruais devido aos ciclos anovulatórios próprios da faixa etária ${ }^{6}$.

Neste estudo, avaliamos os fatores associados à preservação da fertilidade ou dos ovários em mulheres tratadas por massas anexiais em um centro de referência oncológica ginecológica.

\section{Métodos}

Este foi um estudo observacional, parte de uma linha de pesquisa associada ao diagnóstico e tratamento de tumores anexiais e atualmente em andamento no Hospital da Mulher da Universidade Estadual de Campinas (CAISM-UNICAMP). Na presente análise, foram incluídas 206 mulheres com tumores anexiais tratados no CAISM-UNICAMP e que tiveram diagnóstico histológico compatível com benignidade. O estudo foi aprovado pelo Comitê de Ética em Pesquisa da UNICAMP (protocolo 1092/2009) e as pacientes assinaram um o Termo de Consentimento Livre e Esclarecido. Os critérios de exclusão foram mulheres em tratamento para câncer, gestantes e aquelas que já tinham sido submetidas a ooforectomia bilateral. As cirurgias de diagnóstico/tratamento foram realizadas no CAISM-UNICAMP no período de fevereiro de 2010 a janeiro de 2014.

A análise histopatológica ficou a cargo do Departamento de Anatomia Patológica da Unicamp segundo os critérios de Soslow ${ }^{7}$. A preservação da fertilidade foi definida como tumorectomia ou anexectomia unilateral com preservação do anexo contralateral e do útero em mulheres na pré-menopausa. Nas mulheres na pós-menopausa, foi considerado preservação ovariana quando pelo menos um ovário havia sido mantido. Menopausa foi definida como ausência de menstruação por período igual ou maior a 12 meses. Mulheres com histerectomia prévia após os 50 anos de idade foram consideradas menopausadas.

\section{Processamento e análise de dados}

Os dados foram tabulados em planilhas eletrônicas no programa Excel. As análises estatísticas foram feitas utilizando-se o programa R Environment for Statistical Computing ${ }^{8}$. A utilização dos tipos de via de abordagem (laparotomia ou laparoscopia) foi comparada em função dos fatores clínicos e epidemiológicos através de $O d d s$ Ratios (OR) e seus respectivos IC95\%. Foram calculados $\chi^{2}$ para tendências a fim de comparar a associação dos fatores 
Tabela 1. Tipo histológico dos tumores anexiais e estado menopausal

\begin{tabular}{|c|c|c|}
\hline \multirow{3}{*}{ Tumores anexiais } & \multicolumn{2}{|c|}{ Estado menopausal } \\
\hline & Pré-menopausa & Pós-menopausa \\
\hline & n (\%) & n (\%) \\
\hline \multicolumn{3}{|l|}{ Ovariano neoplásico } \\
\hline \multicolumn{3}{|l|}{ Epitelial } \\
\hline $\begin{array}{l}\text { Cistoadenoma seroso ou } \\
\text { cistoadenofibroma seroso }\end{array}$ & $16(52)$ & $20(57)$ \\
\hline Cistoadenoma mucinoso & $13(42)$ & $11(31)$ \\
\hline Tumor de Brenner & $2(6)$ & $2(6)$ \\
\hline $\begin{array}{l}\text { Tumor de Brenner + cistadenoma } \\
\text { mucionoso }\end{array}$ & $0(0)$ & $2(6)$ \\
\hline \multicolumn{3}{|l|}{ Cordão sexual e estroma } \\
\hline Fibroma & $9(82)$ & $25(93)$ \\
\hline Fibrotecoma & $1(9)$ & $0(0)$ \\
\hline Tumor estromal esclerosante & $1(9)$ & $0(0)$ \\
\hline Leiomioma de ovário & $0(0)$ & $2(7)$ \\
\hline \multicolumn{3}{|l|}{ Tumor de células germinativas } \\
\hline Teratoma maduro & $36(100)$ & $8(100)$ \\
\hline \multicolumn{3}{|l|}{ Ovariano não neoplásico } \\
\hline Endometrioma & $17(62)$ & $4(44)$ \\
\hline Cisto funcional & $8(30)$ & $0(0)$ \\
\hline Outros & $2(8)$ & $5(56)$ \\
\hline Extra-ovariano & $15(12)$ & $7(8)$ \\
\hline Total & $120(100)$ & $86(100)$ \\
\hline
\end{tabular}

estudados com o tipo de abordagem cirúrgica, na seguinte sequência: tumorectomia, anexectomia unilateral, anexectomia unilateral com histerectomia e anexectomia bilateral com histerectomia. Foram considerados estatisticamente significantes valores de $\mathrm{p}<0,05$ e calculados IC95\%.

\section{Resultados}

Foram incluídas no estudo 206 mulheres com tumor anexial benigno, sendo que $184(89 \%)$ apresentavam tumores ovarianos e $22(11 \%)$ tumores extra-ovarianos. $\mathrm{Na}$ Tabela 1, observa-se que $120(58 \%)$ pacientes estavam na pré-menopausa e $86(42 \%)$ na pós-menopausa. As neoplasias ovarianas mais frequentes na pré-menopausa foram de células germinativas $36(30 \%)$, seguidas pelas epiteliais 31 (26\%) e do cordão sexual e estroma 11 (9\%). $\mathrm{Na}$ pós-menopausa, houve um predomínio de neoplasias epiteliais $(n=35 ; 41 \%)$, seguidas pelas do cordão sexual e estroma ( $\mathrm{n}=27 ; 31 \%)$ e de células germinativas $(\mathrm{n}=8 ; 9 \%)$. Entre as 36 mulheres com tumores ovarianos não neoplásicos, $21(58 \%)$ apresentavam endometriomas e $8(22 \%)$ cistos funcionais. Das 22 mulheres com tumores extras ovarianos, foram observados 11 (50\%) leiomiomas uterinos, sendo o achado mais frequente na pré e pós-menopausa.

Quando foi avaliada a via de abordagem cirúrgica (Tabela 2), observou-se que a laparoscopia foi mais utilizada em mulheres com $\leq 35$ anos ou menos. A laparotomia
Tabela 2. Via de acesso cirúrgico e características demográficas e histológicas

\begin{tabular}{|c|c|c|c|c|}
\hline \multirow{3}{*}{ Características } & \multicolumn{3}{|c|}{ Via de acesso cirúrgico } & \multirow{3}{*}{ OR (IC95\%) } \\
\hline & Laparoscopia & Laparotomia & $\begin{array}{c}\text { Valor } \\
p\end{array}$ & \\
\hline & $\mathrm{n}(\%)$ & $\mathrm{n}(\%)$ & & \\
\hline \multicolumn{5}{|l|}{ Idade } \\
\hline$\leq 35$ anos & $21(41)$ & $26(17)$ & Ref & \\
\hline 36 a 45 anos & $8(16)$ & $36(23)$ & 0,02 & $3,5(1,3-10,9)$ \\
\hline 46 a 55 anos & $10(20)$ & $37(24)$ & 0,03 & $2,9(1,1-8,3)$ \\
\hline$>55$ anos & $12(23)$ & $56(36)$ & 0,01 & $3,7(1,5-9,7)$ \\
\hline \multicolumn{5}{|l|}{ Paridade } \\
\hline 3 filhos ou mais & $15(30)$ & $76(49)$ & Ref & \\
\hline 1 a 2 filhos & $18(35)$ & $50(32)$ & 0,1 & $0,5(0,2-1,3)$ \\
\hline Nulípara & $18(35)$ & $29(19)$ & $<0,01$ & $0,3(0,1-0,8)$ \\
\hline \multicolumn{5}{|l|}{ Estado menopausal } \\
\hline Pré-Menopausa & $29(57)$ & $83(54)$ & Ref & \\
\hline Pós-Menopausa ${ }^{\#}$ & $22(43)$ & $72(46)$ & 0,7 & $1,1(0,6-2,3)$ \\
\hline $\begin{array}{l}\text { Índice de massa } \\
\text { corpórea }\left(\mathrm{kg} / \mathrm{m}^{2}\right)\end{array}$ & 27,11 & 28,46 & 0,1 & - \\
\hline \multicolumn{5}{|l|}{ Tipo histológico } \\
\hline Neoplasia epitelial & $12(23)$ & $54(35)$ & Ref & \\
\hline $\begin{array}{l}\text { Neoplasia de cordão } \\
\text { sexual e estroma }\end{array}$ & $9(8)$ & $29(19)$ & 0,6 & $0,7(0,2-2,2)$ \\
\hline $\begin{array}{l}\text { Neoplasia de células } \\
\text { germinativas }\end{array}$ & $22(43)$ & $22(14)$ & $<0,01$ & $0,2(0,08-0,6)$ \\
\hline $\begin{array}{l}\text { Ovariano não } \\
\text { neoplásico }\end{array}$ & $6(2)$ & $30(19)$ & 0,8 & $1,1(0,3-3,9)$ \\
\hline Extra-ovariano & $2(4)$ & $20(13)$ & 1,0 & $2,2(0,4-22,0)$ \\
\hline
\end{tabular}

OR: odds ratio; " 11 mulheres na pós-menopausa foram submetidas previamente a histerectomia

foi a via de escolha em pacientes com mais de 55 anos, contrastando com a laparoscopia, que foi a de escolha naquelas mulheres com menos de 35 anos $(\mathrm{p}<0,05)$. Aquelas que tinham três filhos ou mais foram submetidas mais frequentemente a laparotomia em relação às nulíparas $(\mathrm{p}<0,05)$. Não houve diferença significante em relação ao estado menopausal e a via cirúrgica. Quanto ao tipo histológico, apenas os tumores de células germinativas foram mais frequentemente abordados por laparoscopia $(\mathrm{p}<0,01)$. Não houve diferença com relação ao índice de massa corpórea e via de abordagem cirúrgica no presente estudo.

$\mathrm{Na}$ Tabela 3, são descritas as cirurgias realizadas em função das características clínicas, tipo histológico e via de abordagem cirúrgica. Observou-se que, das 46 pacientes com $\leq 35$ anos, 26 (57\%) foram submetidas à tumorectomia e $18(39 \%)$ a anexectomia unilateral com preservação do útero. Apenas em uma paciente com menos de 35 anos $(2,1 \%)$ não houve preservação da fertilidade. Entre as 43 mulheres com idade entre 36 e 45 anos, 5 (11,6\%) foram submetidas a tumorectomia e $22(51,1 \%)$ a anexectomia unilateral com preservação do útero. Entretanto, $16(37,1 \%)$ foram submetidas à retirada do útero, sendo 


\begin{tabular}{|c|c|c|c|c|c|}
\hline \multirow[b]{2}{*}{ Características } & \multicolumn{5}{|c|}{ Cirurgia } \\
\hline & $\begin{array}{l}\text { Tumorectomia } \\
\text { n (\%) }\end{array}$ & $\begin{array}{l}\text { Anexectomia unilateral } \\
\qquad \mathrm{n}(\%)\end{array}$ & $\begin{array}{c}\text { Anexectomia unilateral com } \\
\text { histerectomia } \\
\text { n (\%) }\end{array}$ & $\begin{array}{c}\text { Anexectomia bilateral } \\
\text { com histerectomia } \\
\text { n (\%) }\end{array}$ & Valor $\mathrm{p}^{*}$ \\
\hline \multicolumn{6}{|l|}{ Idade } \\
\hline$\leq 35$ anos & $26(57)$ & $18(39)$ & $1(2)$ & $1(2)$ & Ref \\
\hline 36 a 45 anos & $5(12)$ & $22(51)$ & $7(16)$ & $9(21)$ & $<0,01$ \\
\hline \multicolumn{6}{|l|}{ Paridade } \\
\hline 3 filhos ou mais & $3(3)$ & $24(27)$ & $10(11)$ & $53(59)$ & Ref \\
\hline 1 a 2 filhos & $9(13)$ & $23(34)$ & $5(7)$ & $31(46)$ & 0,02 \\
\hline Nulipara & $20(43)$ & $12(26)$ & $4(9)$ & $10(22)$ & $<0,01$ \\
\hline \multicolumn{6}{|l|}{ Estado menopausal } \\
\hline \multicolumn{6}{|l|}{ Tipo histológico } \\
\hline Neoplasia epitelial & $5(8)$ & $21(32)$ & $6(9)$ & $34(51)$ & Ref \\
\hline Neoplasia de cordão sexual e estroma & $0(0)$ & $12(32)$ & $3(8)$ & $23(60)$ & 0,2 \\
\hline Neoplasia de células germinativas & $17(39)$ & $19(43)$ & $1(2)$ & $7(16)$ & $<0,01$ \\
\hline Ovariano não neoplásico & $7(19)$ & $5(14)$ & $4(11)$ & $20(56)$ & 0,9 \\
\hline Extra-ovariano & $3(15)$ & $2(10)$ & $5(25)$ & $10(50)$ & 0,8 \\
\hline \multicolumn{6}{|l|}{ Via de acesso cirúrgico } \\
\hline Laparoscopia & $16(31)$ & $22(43)$ & $2(4)$ & $11(22)$ & Ref \\
\hline Laparotomia & $16(11)$ & $37(24)$ & $17(11)$ & $83(54)$ & $<0,01$ \\
\hline
\end{tabular}

Obs: Porcentagens calculadas na linha. ${ }^{*} 11$ mulheres na pós-menopausa foram submetidas previamente a histerectomia; *Teste de proporções progressivas; Total de 204 pacientes, 2 excluídas por apenas biópsia e outra por exérese de colo restante.

$7(16,2 \%)$ com anexectomia unilateral e $9(20,9 \%) \mathrm{com}$ anexectomia bilateral. Entre as 47 mulheres com 46 a 55 anos, $30(63,8 \%)$ foram submetidas à anexectomia bilateral com histerectomia. Nas 68 mulheres com mais de 55 anos, a anexectomia bilateral com histerectomia foi realizada na maioria $54(79,4 \%)$. Com relação ao estado menopausal, 26 (28\%) das pacientes submetidas à histerectomia com anexectomia bilateral estavam na pré-menopausa. Mulheres $\leq 35$ anos foram mais frequentemente operadas por laparoscopia e a laparoscopia esteve associada a maior taxa de preservação de fertilidade quando comparada com a laparotomia $(\mathrm{p}<0,01)$. Nas cirurgias realizadas por laparotomia, em $54,2 \%$ foi realizada histerectomia com anexectomia bilateral e apenas em 10,4\% foi realizada tumorectomia. Já nas cirurgias realizadas por via laparoscópica, em 16 (31,3\%) foi realizada tumorectomia. A maioria das pacientes submetidas à tumorectomia $(\mathrm{n}=17 ; 53 \%)$ possuia tumores de células germinativas.

\section{Discussão}

Embora haja um consenso na literatura sobre a importância da preservação dos ovários no menacme, neste estudo verificamos que um número elevado de mulheres no menacme ainda é submetida à ooforectomia bilateral para o tratamento de tumores benignos de ovário. Muitas mulheres são submetidas à anexectomia ao invés de tumorectomia, o que poderia inviabilizar a preservação da fertilidade e dos ovários no caso de tumores bilaterais e assincrônicos.

Entre as mulheres que se encontravam no menacme, houve um predomínio de tumores de células germinativas (os teratomas maduros) enquanto na pós menopausa foram encontrados mais tumores epiteliais, sendo os mais frequentes os cistadenomas serosos, o que está de acordo com a literatura ${ }^{4}$.

Quanto à abordagem cirúrgica dos tumores anexiais benignos, na mulher jovem a cirurgia preconizada é a tumorectomia com preservação de parênquima ovariano. O tecido cortical remanescente, mesmo escasso, contém folículos que irão suprir as funções hormonais e de fertilidade 9 . A tumorectomia ou cistectomia por laparoscopia é segura para tumores ou cistos de até $10 \mathrm{~cm}$, considerando-se um risco de ruptura de cisto de 12 a $18 \%$ com baixa incidência de peritonite, $0,2 \%{ }^{10}$.

Observou-se que $39 \%$ de mulheres com $\leq 35$ anos de idade foram submetidas à anexectomia unilateral ao invés de tumorectomia unilateral, possivelmente por não se conseguir individualizar parênquima ovariano normal, 
sobretudo no caso de tumores volumosos. No entanto, em um estudo envolvendo 382 pacientes com teratomas benignos e tratadas por cirurgia conservadora, a taxa de recorrência foi de 4,2\%, sendo os fatores de risco: idade menor do que 30 anos, tumores maiores que $8 \mathrm{~cm} \mathrm{e}$ tumores bilaterais (dados que reforçam a necessidade de conservação dos ovários nas mulheres mais jovens $)^{11}$.

Quanto à via de abordagem cirúrgica em nosso estudo, observamos predomínio de laparotomias $(75,2 \%)$ em relação a laparoscopias $(24,8 \%)$, sendo que a laparoscopia foi mais frequentemente realizada nas pacientes com $\leq 35$ anos. A laparoscopia também foi mais utilizada em pacientes jovens em um estudo que avaliou 96.014 laparoscopias por doença benigna ovariana. Houve, porém um predomínio acentuado em relação às laparotomias, realizadas em 39.779 mulheres. A laparoscopia é considerada um procedimento seguro para o tratamento de tumores benignos de ovário ${ }^{12}$.

Os dados na literatura são escassos quanto à realização de histerectomia no momento do tratamento de lesão ovariana benigna. Entre os casos por nós estudados, $37,1 \%$ das mulheres entre 36 a 45 anos foram submetidas à histerectomia, ou seja, não tiveram a fertilidade preservada. Ao se realizar a histerectomia total haveria a eliminação do risco para cânceres de endométrio e colo uterino, além da simplificação do uso da terapia hormonal (só com estrogênios). Mas os casos de sangramento uterino aumentado na perimenopausa devem ser investigados por biópsia de Pipelle, a fim de excluir doenças endometriais, antes de se realizar uma histerectomia "profilática"13.

Por outro lado, nos casos de mulheres que não desejam mais filhos, ao se realizar a histerectomia devem ser pesadas complicações que aumentam a morbidade peri e pós operatória, como hemorragia, lesões de órgãos adjacentes (tratos gastrointestinal e gênito-urinário), eventos tromboembólicos e infecção ${ }^{14}$. Em nosso estudo, das $68 \mathrm{mu}-$ lheres com mais de 55 anos, $10 \%$ não foram submetidas à histerectomia, provavelmente pelo alto risco cirúrgico.

Quanto às razões para se realizar a histerectomia subtotal, destacam-se abreviar o tempo cirúrgico, menor perda sanguínea, menos infecções, o não prejuízo da função sexual (esta última ainda sem comprovação científica). No entanto, deve-se considerar o risco de câncer de colo residual, cuja ocorrência é rara ( $2 \%$ dos cânceres de colo), mas não desprezível e, por conseguinte, a necessidade de manter o rastreamento para este tipo de neoplasia ${ }^{15}$. Assim, com o treinamento adequado dos ginecologistas, a técnica da histerectomia total deve ser priorizada e não apresenta mais complicações do que a subtotal ${ }^{15}$.

A ooforectomia cirúrgica, tanto na pré quanto na pós-menopausa, está relacionada a riscos mais elevados de doenças cardiovasculares, osteoporose, fraturas de quadril, demência, doença de Parkinson, depressão, ansiedade e disfunção sexual, segundo revisão realizada por Parker em $2010^{16}$. Mesmo após a menopausa, o ovário continua a produzir androstenediona e testosterona que são convertidos em estrona no tecido adiposo periférico, músculo e pele ${ }^{17}$.

Apesar do estudo de Parker et al. ${ }^{18}$ baseado no modelo analítico de Markov demonstrar maior mortalidade e morbidade após ooforectomia em mulheres com menos de 65 anos, a recomendação da American College of Obstetricians and Gynecologists (ACOG) de $2008^{19}$ sugere que a menopausa seja o fator determinante para ooforectomia no momento da histerectomia. Dando suporte a esta recomendação, em estudo recente nos EUA, dois terços dos obstetras-ginecologistas são favoráveis à ooforectomia no momento da histerectomia em mulheres menopausadas, entre 51 a 65 anos $^{20}$.

Quando se opta pela conservação ovariana, seja para a preservação da fertilidade na mulher sem prole definida, seja para a preservação da função ovariana na pré-menopausa, são temidos os riscos de nova abordagem cirúrgica devido à bilateralidade assincrônica do tumor ou risco de câncer de ovário. No entanto, em um estudo observacional envolvendo 13.035 mulheres submetidas à histerectomia por doença benigna com preservação ovariana. uma nova cirurgia por cisto simples foi realizada em $6,8 \%$ das pacientes; e por tumor suspeito de neoplasia, em 2,8\%. Este mesmo estudo indicou que 34 (0,26\%) mulheres morreram por câncer de ovário. Em contrapartida, a cada ano, entre as mulheres americanas, 14.700 morrem por câncer de ovário, enquanto 326.900 morrem por doenças coronarianas ${ }^{6}$.

Concluindo, embora exista uma recomendação em realizar apenas tumorectomia em mulheres com $\leq 35$ anos, uma proporção significativa ainda é submetida à anexectomia para o tratamento dos tumores de ovário benignos. No presente estudo, em mulheres entre 36 e 45 anos, apenas dois terços tiveram sua fertilidade preservada e $20 \%$ tiveram ambos os ovários removidos, o que é considerado um número alto, já que dentro desta faixa etária, os riscos de menopausa cirúrgica superam os benefícios.

\section{Contribuição dos autores}

ES, AY e HP participaram da coleta e sistematização dos casos. ES, AY prepararam o artigo sob supervisão de SD e LOS, que desenharam o estudo e fizeram a análise estatística. LAA contribuiu com o suporte na especialidade de Anatomia Patológica.

\section{Agradecimentos}

Este estudo foi parcialmente financiado pelo Auxílio Fapesp 2012/15059-8. 
1. Curtin JP. Management of the adnexal mass. Gynecol Oncol. 1994;55(3 Pt 2):S42-6.

2. Sethi D, Ahluvalia C, Sharma U, Khetarpal S. A synchronous presentation of two different ovarian tumors: a rare occurrence. Ann Med Health Sci Res. 2013;3(2):268-70.

3. Kim M, Kim NY, Lee DY, Yoon BK, Choi D. Clinical characteristics of ovarian teratoma: age-focused retrospective analysis of 580 cases. Am J Obstet Gynecol. 2011 ;205(1):32.e1-4.

4. Kurman RJ, Ellenson LH, Ronnett BM, editors. Blaustein's pathology of the female genital tract. 6th ed. New York: Springer; 2011.

5. Shuster LT, Gostout BS, Grossardt BR, Rocca WA. Prophylactic oophorectomy in premenopausal women and long-term health. Menopause Int. 2008; 14(3):111-6.

6. Parker WH, Broder MS, Chang E, Feskanich D, Farquhar C, Liu Z, et al. Ovarian conservation at the time of hysterectomy and longterm health outcomes in the nurse's health study. Obstet Gynecol. 2009; $113(5): 1027-37$.

7. Soslow RA. Histologic subtypes of ovarian carcinoma: an overview. Int J Gynecol Pathol. 2008;27(2):161-74.

8. R Core Team. The R project for statistical computing [Internet]. Vienna: R Foundation for Statistical Computing; 2014 [cited 2014 Ago 10]. Available from: <http://www.R-project.org/>

9. Gobbi D, Fascetti Leon F, Aquino A, Melchionda F, Lima M. Metachronous bilateral ovarian teratoma: a germ-line familial disorder and review of surgical management options. J Pediatr Adolesc Gynecol. 2013;26(5):e105-7.

10. Medeiros LR, Rosa DD, Bozzetti MC, Fachel JM, Furness S, Garry $R$, et al. Laparoscopic versus laparotomy for benign ovarian tumour. Cochrane Database Syst Rev. 2009;(2):CD004751.
11. Harada M, Osuga Y, Fujimoto A, Fujimoto A, Fujii T, Yano T, et al. Predictive factors for recurrence of ovarian mature cystic teratomas after surgical excision. Eur J Obstet Gynecol Reprod Biol. 2013;171 (2):325-8.

12. Wu MP, Wu CJ, Long $\mathrm{CY}, \mathrm{Ho} \mathrm{CH}$, Huang $\mathrm{KH}$, Chu $\mathrm{CC}$, et al. Surgical trends for benign ovarian tumors among hospitals of different accreditation levels: an 11 -year nationwide populationbased descriptive study in Taiwan. Taiwan J Obstet Gynecol. 2013;52(4):498-504.

13. Sweet MG, Schmidt-Dalton TA, Weiss PM, Madsen KP. Evaluation and management of abnormal uterine bleeding in premenopausal women. Am Fam Physician. 2012;85(1):35-43.

14. Clarke-Pearson DL, Geller EJ. Complications of hysterectomy. Obstet Gynecol. 2013;121(3):654-73.

15. Hellstrom AC, Hellman K, Pettersson BF, Andersson S. Carcinoma of the cervical stump: fifty years of experience. Oncol Rep. $2011 ; 25(6): 1651-4$.

16. Parker WH. Bilateral oophorectomy versus ovarian conservation: effects on long-term women's health. J Minim Invasive Gynecol. 2010;17(2):161-6.

17. Sluijmer AV, Heineman M, De Jong FH, Evers JL. Endocrine activity of the postmenopausal ovary: the effects of pituitary down-regulation and oophorectomy. J Clin Endocrinol Metab. 1995;80(7):2163-7.

18. Parker WH, Broder MS, Liu Z, Shoupe D, Farquhar C, Berek JS. Ovarian conservation at the time of hysterectomy for benign disease. Obstet Gynecol. 2005; 106(2):219-26.

19. ACOG. ACOG Practice Bulletin No. 89. Elective and risk-reducing salpingo-oophorectomy. Obstet Gynecol. 2008; 111 (1):231-41.

20. Harmanli $O$, Shinnick J, Jones K, St Marie P. Obstetrician-gynecologists' opinions on elective bilateral oophorectomy at the time of hysterectomy in the United States: a nationwide survey. Menopause. 2014;21 (4):355-60. 